\title{
Sharing Social Networks Using a Novel Differentially Private Graph Model
}

\author{
Tianchong Gao*, Feng $\mathrm{Li}^{*}$ \\ *Indiana University-Purdue University Indianapolis, Indianapolis, IN, U.S.A. \\ tgao@iupui.edu, fengli@iupui.edu
}

\begin{abstract}
Online social networks (OSNs) often contain sensitive information about individuals. Therefore, anonymizing social network data before releasing it becomes an important issue. Recent research introduces several graph abstraction models to extract graph features and add sufficient noise to achieve differential privacy.

In this paper, we design and analyze a comprehensive differentially private graph model that combines the dK-1, dK2 , and $\mathrm{dK}-3$ series together. The $\mathrm{dK}-1$ series stores the degree frequency, the dK-2 series adds the joint degree frequency, and the $\mathrm{dK}-3$ series contains the linking information between edges. In our scheme, low dimensional data makes the regeneration process more executable and effective, while high dimensional data preserves additional utility of the graph. As the higher dimensional model is more sensitive to the noise, we carefully design the executing sequence. The final released graph increases the graph utility under differential privacy.
\end{abstract}

Index terms-Social network data publishing; anonymization; differential privacy; dK graph abstraction model

\section{INTRODUCTION}

Studying OSNs through graph analysis could produce knowledge of human social relationships, help feed advertisements to recommendation targets, and evaluate the effectiveness of applications. Since OSN data contains personal information, any releasing procedure without sufficient anonymization work causes panic to the users of social media. Various anonymization techniques have been proposed. Differential privacy is one of the most remarkable techniques, since it could theoretically achieve a strong privacy guarantee [2].

Differential privacy requires graph abstraction models to convert the graph structure into numerical-like data. Fig. 1 gives an example of the $\mathrm{dK}$ model. The $\mathrm{dK}$ model is separated into different dimensions. The $\mathrm{dK}-\mathrm{N}$ model captures the degree distribution of connected components of size N. For example, $\langle 1,4\rangle=2$ means that there are two node groups of degree 1 and degree 4. Sala et al. employed the dK-2 series as the graph abstraction model to achieve differential privacy [10].

However, deploying one abstraction model can only capture some aspects of information, while other utilities are lost in the published graph. For example, because the dK2 graph model is the record of edges, it may not preserve information involving more than two nodes, e.g., the clustering coefficient. The limitations in the models restrict their ability to achieve structural similarity under differential privacy. Therefore, choosing the right abstraction model becomes an important issue. Mahadevan et al. proved that $\mathrm{dK}$ models in higher dimensions have more information than the ones in lower dimensions, e.g., the $\mathrm{dK}-3$ model is more precise than the $\mathrm{dK}-2$ model [5].

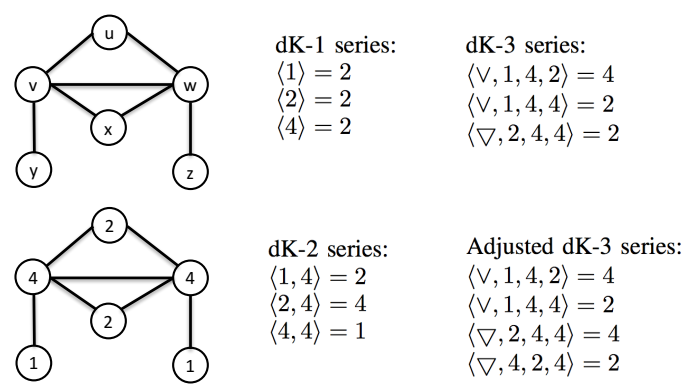

Fig. 1: An example of the $\mathrm{dK}$ model

Our initial idea is to preserve differential privacy with the $\mathrm{dK}-3$ model. To the best of our knowledge, there is no systematic regeneration algorithm for $\mathrm{dK}-3$ model because of its complexity $[5,10]$. In our study, we also find that it is hard to reconstruct the graph with only the $\mathrm{dK}-3$ series. However, after studying the differences between the $\mathrm{dK}-1, \mathrm{dK}-2$, and $\mathrm{dK}-3$ series, we find that low dimension $\mathrm{dK}$ series, i.e., $\mathrm{dK}$ 1 and $\mathrm{dK}-2$, can help the regeneration process. And we can use some rewiring algorithms to inject the $\mathrm{dK}-3$ series in our published graph.

In this paper, we absorb the benefits of different models and design a new comprehensive model that combines three levels of $\mathrm{dK}$ graph models together. To achieve differential privacy, we introduce noise on the $\mathrm{dK}-2$ level, which causes less distortion than on $\mathrm{dK}-3$ level. Then we use the perturbed $\mathrm{dK}-2$ series to get the corresponding $\mathrm{dK}-3$ and $\mathrm{dK}-1$ series. After that, we use three levels of $\mathrm{dK}$ series together in our scheme to construct a new graph. The noise impact is the major challenge in the graph regeneration process. Although the three models in our scheme are closely related, they may conflict with each other because of noise. Hence, we first use some $\mathrm{dK}$ information to regenerate an intermediate graph, then use the remaining information to rewire the edges.

The major technical contributions are the following: (1) We are the first to build the systematic regeneration algorithm for embedding dK-3 information in graph anonymization, which helps to preserve more utility than existing dK models. (2) We combine the $\mathrm{dK}-3$ model with both $\mathrm{dK}-1$ and $\mathrm{dK}-2$ models in sampling and graph regeneration, which mitigates the high sensitivity and complexity in the $\mathrm{dK}-3$ model and makes the design practical. (3) We design two different routes, CAT and LTH, to generate the graph efficiently and effectively, even under the noise impact. (4) We reveal the insights and challenges of using different levels of $\mathrm{dK}$ abstraction models jointly to enhance the utility under differential privacy. 


\section{PRELIMINARIES}

\section{A. The dK graph model}

In this paper, an OSN graph is modeled as an undirected graph $G=(V, E)$, where $V$ is the set of vertices and $E$ is the set of edges. $|V|$ means the cardinality of set $V . d_{v}$ is the degree of the vertex $v . e_{u, v}$ means an edge between nodes $u$ and $v$.

Since differential privacy is applied on the query result, the $\mathrm{dK}$ graph model is chosen to transform an input graph into a set of structural statistics. Although many models can give graph statistical information, the $\mathrm{dK}$ graph model is better than most of them because the $\mathrm{dK}$ series could be used to construct a new graph having structural similarities with the original graph.

The dK-N model captures the degree distribution of connected components of size $\mathrm{N}$ in a target graph [5]. For example, dK-1, also known as the node degree distribution, counts the number of nodes in each degree value. The $\mathrm{dK}$ 2 model, also called joint degree distribution, captures the number of edges in each combination of two degree values. In this paper, we define the dimension of $\mathrm{dK}$ information as the subgraph size $(\mathrm{N})$. Hence, the $\mathrm{dK}-1$ series has lower dimension than $\mathrm{dK}-2$.

The dK-3 model captures the number of 3-node subgraphs with different combinations of node degrees. Specifically, there are two kinds of 3-node subgraphs: wedges and triangles.

The wedge dK-3 entry: The $\mathrm{dK}-3$ entry $\left\langle\vee, d_{u}, d_{v}, d_{w}\right\rangle=$ $k$ means that there are $k 3$-node wedges which have the node degree values equal to $d_{u}, d_{v}$, and $d_{w}$, and each of the two subgraphs have at least one different node. In order to prevent double counting, $d_{u}$ should be less than or equal to $d_{w}$. Assume the combination of nodes $u, v$ and $w$ forms such a subgraph, then $w$ should not be the neighbor of $u$.

The triangle dK-3 entry: The dK-3 entry $\left\langle\nabla, d_{u}, d_{v}, d_{w}\right\rangle=k$ means that there are $k$ triangles with node degree $d_{u}, d_{v}$, and $d_{w}$. To prevent double counting, we have $d_{u} \leqslant d_{v} \leqslant d_{w}$. The node set of the subgraph should be $V=\{u\} \cup\left\{v \mid e_{u, v} \in E\right\} \cup\left\{w \mid e_{v, w} \in E \wedge e_{u, w} \in E\right\}$.

The error between two dK-3 series is defined as the sum of all absolute differences in each corresponding dK-3 entry.

$$
\operatorname{err}_{3}=\sum_{\mathrm{dK}-3}\left|k_{i}-k_{i}^{\prime}\right| .
$$

Similarly, $e r r_{1}$ and $e r r_{2}$ measure the errors in the $\mathrm{dK}-1$ and $\mathrm{dK}-2$ series. Our purpose here is to reduce these errors to decrease unnecessary noise.

\section{SCHEME}

Given an OSN, our goal is to publish an anonymized network that preserves the structural utility as much as possible while satisfying $\epsilon$-differential privacy. The general idea is to add sufficient noise to the $\mathrm{dK}$ model and reconstruct a graph $G$ based on the perturbed dK series.

As mentioned in previous research, a model of higher dimension is more precise, but it is difficult to directly reconstruct the graph from the $\mathrm{dK}-3$ series $[5,10]$. It is not a good idea to start with adding noise to the dK-3 series. Another

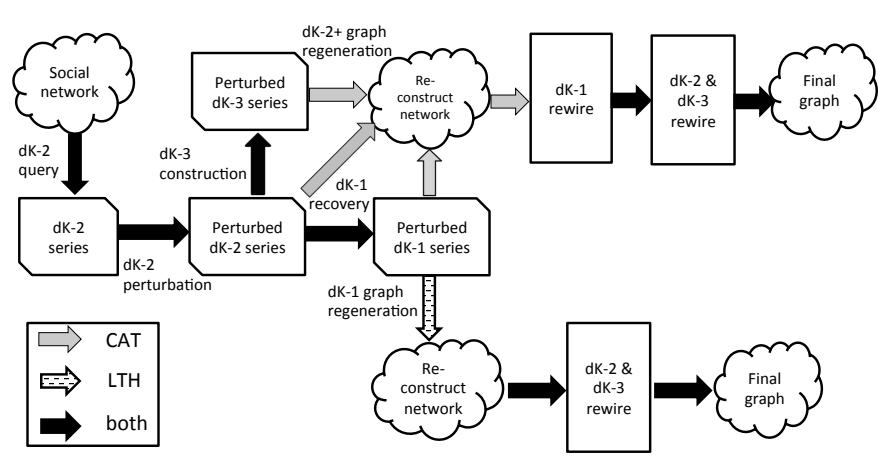

Fig. 2: Scheme overview

option is to add noise to the $\mathrm{dK}-1$ series. However, the $\mathrm{dK}-2$ and $\mathrm{dK}-3$ series also need the corresponding perturbation in order to maintain consistency. Because the $\mathrm{dK}-1$ series has no information of edges, it is hard to do those perturbations. In the scheme, we inject noise into the $\mathrm{dK}-2$ series.

After injecting noise, our purpose in the graph regeneration process is to publish a graph with similar $\mathrm{dK}$ series as the perturbed results (in all three levels). There are two main routes in graph regeneration, starting with the $\mathrm{dK}-1$ series or starting with the dK-2 series. We design two sub-schemes, shown in Fig.2, called CAT and LTH, and the mutual steps are marked in 'both'. After the regeneration part, both subschemes have an active rewiring procedure to mitigate their errors, e.g., the $\mathrm{dK}-2$ and $\mathrm{dK}-3$ series have not been used by LTH.

In the following sections, we discuss these components, which are also shown in Fig. 2:

1. Perturb the dK-2 series under differential privacy,

2. Build the $\mathrm{dK}-3$ model with perturbed $\mathrm{dK}-2$ series,

3. Recover the dK-1 information,

4. Reconstruct the perturbed graph with different combinations of $\mathrm{dK}$ series,

\section{A. $d K-2$ perturbation}

After counting the $\mathrm{dK}-2$ entries, we apply the Laplace mechanism to achieve differential privacy [3].

Theorem 1 (LAPLACE MECHANISM). For a function $f$ : $\mathcal{D} \rightarrow \mathbb{R}^{d}$, the randomized algorithm $\mathcal{A}$

$$
\mathcal{A}(G)=f(G)+\operatorname{Lap}\left(\frac{\triangle f}{\epsilon}\right)
$$

achieves $\epsilon$-differential privacy [6]. The sensitivity $\triangle f$ of a function $f$ is the maximum distance of any two neighbor databases $D_{1}$ and $D_{2}$ in $\ell_{1}$ norm.

According to Equation 2, the noise level is determined by the sensitivity $\triangle f$ and the privacy parameter $\epsilon$. In OSN anonymization problem, the sensitivity shows the impact of adding or deleting an edge in the model. The sensitivity of $\mathrm{dK}-2$ series is shown below.

Property 1. Given an entry $\left\langle d_{x}, d_{y}\right\rangle$ in the $d K-2$ model, the sensitivity $\triangle f$ is upper bounded by $2 \cdot d_{x}+2 \cdot d_{y}+1$.

Proof. Let $e_{x, y}$ be a new edge added to the graph $G$ between nodes $x$ and $y$. There is one new dK-2 series $\left\langle d_{x}, d_{y}\right\rangle$ getting 
incremented by 1 . Also, the degrees of $x$ and $y$ increase from $d_{x}$ and $d_{y}$ to $d_{x}+1$ and $d_{y}+1$, respectively. In the original $\mathrm{dK}-$ 2 model, there are $d_{x}$ series related with the node $x$, which are in the form of $\left\langle d_{u}, d_{x}\right\rangle$ and $\left\langle d_{x}, d_{u}\right\rangle$. These series are deleted, while new series, $\left\langle d_{u}, d_{x}+1\right\rangle$ and $\left\langle d_{x}+1, d_{u}\right\rangle$, are added. Hence, totally $2 \cdot d_{x}+2 \cdot d_{y}+1 \mathrm{dK}-2$ series are changed when the new edge is added.

Hence, we set $\triangle f=2 \cdot d_{x}+2 \cdot d_{y}+1$ in our scheme. The perturbed dK-2 entry is $\left\langle d_{x}, d_{y}\right\rangle=k+\operatorname{Lap}\left(\frac{\triangle f}{\epsilon}\right)$.

\section{B. $d K-3$ construction}

Given the $\mathrm{dK}-2$ model, we construct the $\mathrm{dK}-3$ model to preserve edge linking information. Particularly, if one $\mathrm{dK}-2$ entry is perturbed, its corresponding $\mathrm{dK}-3$ entries are also perturbed, which leaks no edge information beyond differential privacy. Hence, we examine the influence of $\mathrm{dK}-2$ perturbation on the dK-3 model in the example of one edge $e_{u, v}$, then do the modification.

First, there is a simple case in which all three-node pairs in the graph are wedges. There are $d_{u}-1$ edges connected with the node $u$. Then the edge produces $d_{u}-1 \mathrm{dK}-3$ entries in the form of $\left\langle\vee, d_{x}, d_{u}, d_{v}\right\rangle$ or $\left\langle\vee, d_{v}, d_{u}, d_{x}\right\rangle$. Similarly, it also produces $d_{v}-1 \mathrm{dK}-3$ entries in the form of $\left\langle\vee, d_{y}, d_{v}, d_{u}\right\rangle$ or $\left\langle\vee, d_{u}, d_{v}, d_{y}\right\rangle$. Hence, there are totally $d_{u}+d_{v}-2 \mathrm{dK}-3$ wedges entries produced by the edge $e_{u, v}$.

Second, we improve the case that the graph has some triangles. Adding an edge $e_{u, v}$ between node $u$ and $v$, if they have a common neighbor $x$, the original entry $\left\langle\vee, d_{u}, d_{x}, d_{v}\right\rangle$ will be changed to $\left\langle\nabla, d_{u}, d_{x}, d_{v}\right\rangle$. However, if they do not have a common neighbor, there will be some new entries added, like the case before. Therefore, the total number of $\mathrm{dK}-3$ entries containing the edge $e_{u, v}$ is also affected by the number of triangles.

Adjusted dK-3 model. We find that if we deploy some specific counting method for triangles, the wedges and triangles can be treated equally. Thus, the adjusted dK-3 model is proposed to simplify the calculation of the $\mathrm{dK}-3$ series. The adjusted model is completely based on the basic $\mathrm{dK}-3$ series. Using the adjusted model will not increase or decrease the ability of the $\mathrm{dK}-3$ series to present or reconstruct the graph. The new model does not change the wedge entry $\left\langle\vee, d_{u}, d_{v}, d_{w}\right\rangle$. But if there is a triangle entry $\left\langle\nabla, d_{u}, d_{v}, d_{w}\right\rangle=k$, it will be replaced by three entries, $\left\langle\nabla, d_{u}, d_{v}, d_{w}\right\rangle=k,\left\langle\nabla, d_{v}, d_{w}, d_{u}\right\rangle=k$, and $\left\langle\nabla, d_{w}, d_{u}, d_{v}\right\rangle=k$. In the following sections, all $\mathrm{dK}-3$ series are sampled in the adjusted $\mathrm{dK}-3$ model. After deploying the adjusted dK-3 model, deleting or adding an edge $e_{u, v}$ always changes $d_{u}+d_{v}-2 \mathrm{dK}-3$ entries. In the following sections, a wildcard character $*$ is used to match $\vee$ and $\nabla$. The $\mathrm{dK}-3$ entry is like $\left\langle *, d_{u}, d_{v}, d_{w}\right\rangle$.

In the above section, the $\mathrm{dK}-2$ series is perturbed for privacy. Each unit of increment or decrement in $\mathrm{dK}-2$ entries could be viewed as one edge adding or deleting. Then we do corresponding modifications on the $\mathrm{dK}-3$ series. Specifically, for increasing or decreasing, there are three possible changes in $\mathrm{dK}-3$ entries

1) Replacement: If $\left\langle d_{u}, d_{v}\right\rangle$ decreased by one and $\left\langle d_{u}, d_{w}\right\rangle$ increased by one, the graph replaces the edge $e_{u, v}$ by

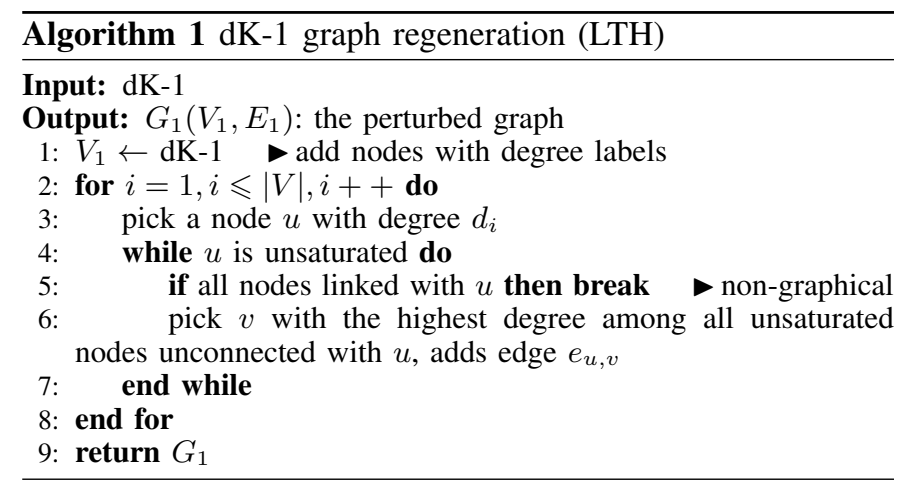

$e_{u, w}$. So we pick $\min \left(d_{w}, d_{v}\right)+d_{u}-2 \mathrm{dK}-3$ entries and use the number $d_{w}$ to replace $d_{v}$ in the $\mathrm{dK}-3$ entries.

2) Subtracting: For each unit of decrement in $\left\langle d_{u}, d_{v}\right\rangle$, the graph deletes the edge $e_{u, v}$. So we reduce the dK-3 entries containing $\left\langle d_{u}, d_{v}\right\rangle$ by the total value of $d_{u}+d_{v}-2$.

3) Adding: For each unit of increment in $\left\langle d_{u}, d_{v}\right\rangle$, the graph adds an edge $e_{u, v}$. The formation part is a little special because there is no original record of the neighbors of $u$ or $v$. So we randomly pick a structure, wedge or triangle, and a degree number, $d_{x}$, in the range of $\left[1, d_{\max }\right]$. Then we add the total value of $d_{u}+d_{v}-2$ to the $\mathrm{dK}-3$ entries containing $\left\langle d_{u}, d_{v}, d_{x}\right\rangle$.

\section{C. $d K-1$ recovery}

The $\mathrm{dK}-1$ series is also important in the generation of the graph. Unlike the $\mathrm{dK}-3$ series, it can be recovered directly from the $\mathrm{dK}-2$ series. It is calculated by the following equation.

$$
\left\langle d_{v}\right\rangle=\frac{\sum_{\mathrm{dK}-2 \text { entry }}\left\langle d_{u}, d_{v}\right\rangle+\sum_{\mathrm{dK}-2 \text { entry }}\left\langle d_{v}, d_{u}\right\rangle}{d_{v}} .
$$

Equation 3 shows that the high dimensional data, e.g., dK-2, contains all the information of the low dimensional data, e.g., $\mathrm{dK}-1$.

\section{Graph regeneration}

Given the target $\mathrm{dK}-2, \mathrm{dK}-3$, and $\mathrm{dK}-1$ series, we need to regenerate the corresponding graph. Focusing on a different level of $\mathrm{dK}$ series, we propose two sub-schemes, namely CAT and LTH, with different regeneration algorithms.

The LTH scheme starts from the $\mathrm{dK}-1$ series, the main reason is that $\mathrm{dK}-1$ series is the base of the graph. If the degree of a node has an error, there will be large distortion on the corresponding dK-2 and dK-3 series. Hence, LTH just needs the $\mathrm{dK}-1$ information and to generate a graph with the least $e r r_{1}$. By contrast, the CAT scheme considers the dK-2 and $\mathrm{dK}-3$ series in regeneration because rewiring edges cannot guarantee to achieve the lowest err $_{2}$ and err $_{3}$. This scheme aims to reduce $\mathrm{err}_{2}$ the most, while preserving some $\mathrm{dK}-3$ information as well.

In both schemes, we call a node 'saturated' if it has as many neighbors as its label (dK-1 information), and call it 'unsaturated' otherwise. If the value of a dK entry in the graph reaches the target value, we call it 'full'.

LTH Algorithm 1 firstly sorts the degree sequence into a non-increasing order, which means $d_{1} \geqslant d_{2} \geqslant \ldots \geqslant d_{|V|}$. 

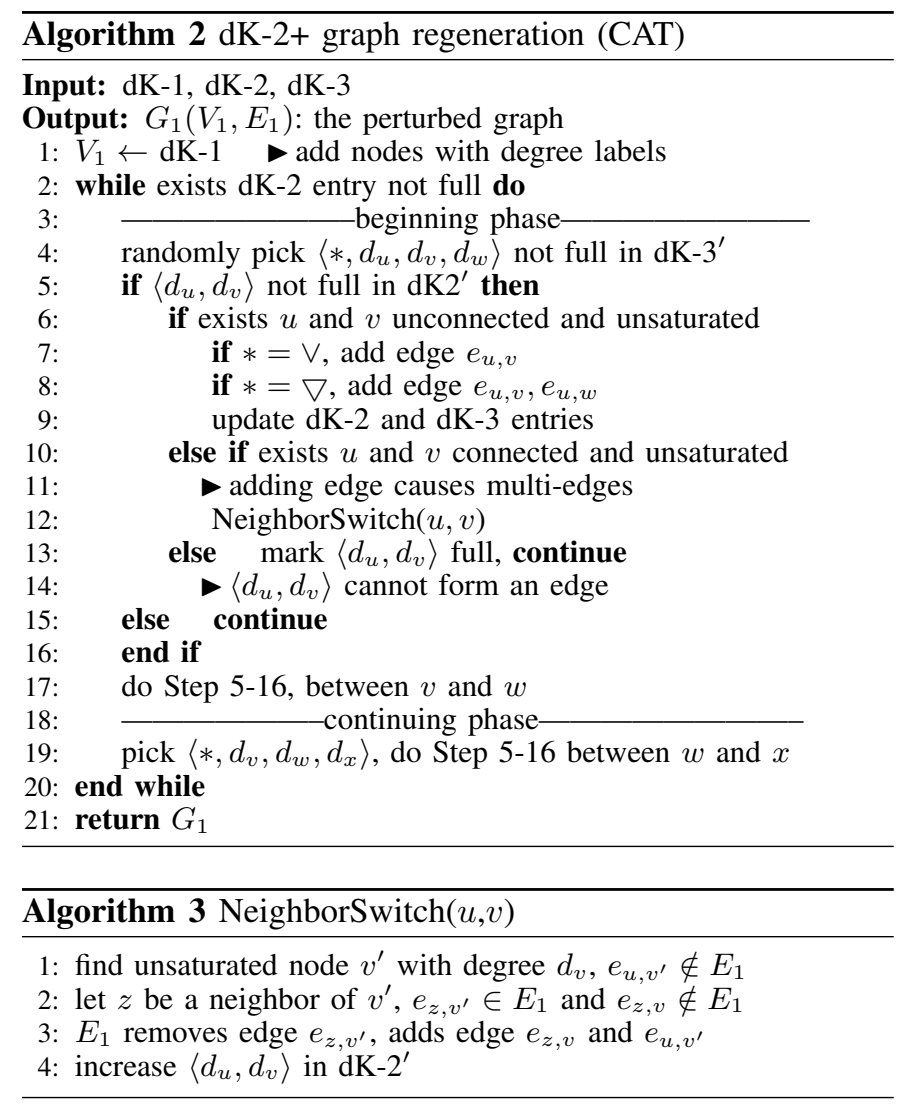

Each number in the sequence also represents the target degree value of a corresponding node. Then, beginning from the first node with degree $d_{1}$, the algorithm links the node with $d_{1}$ nodes. These nodes are chosen from the set of nodes that are unconnected with the first node, which having highest degree values among the set. According to [1], a graph can be reconstructed with the exact $\mathrm{dK}-1$ information if and only if every node $v$ is connected to all $d_{v}$ nodes in the leftmost part of the degree sequence (having the highest degree values).

CAT Algorithm 2 orderly picks dK-3 entries. For example, if it picks $\left\langle *, d_{u}, d_{v}, d_{w}\right\rangle$ in previous round, this round it picks $\left\langle *, d_{v}, d_{w}, d_{x}\right\rangle$. Then it tries to add one edge to the graph if these two nodes can pass the edge check. Here, the edge check means there are two unsaturated nodes with the correct degree, the two nodes are not connected, and the corresponding $\mathrm{dK}$ 2 entry is not full. After adding the edge, the corresponding $\mathrm{dK}-2$ and $\mathrm{dK}-3$ entries are updated. The regeneration process stops when there are no node pairs that can pass the edge check. Also, in the edge check process, it may happen that the only pair of unsaturated nodes are already connected. Simply connecting them together forms multi-edges in the graph, which is forbidden in OSNs. Algorithm 3 switches one neighbor from a saturated node to an unsaturated node with the same label.

The orderly picking is the continuing phase in Algorithm 2. However, if Algorithm 2 cannot find the continuing $\mathrm{dK}$ 3 series, e.g., $\left\langle *, d_{v}, d_{w}, d_{x}\right\rangle$, it uses the beginning phase to randomly choose a new $\mathrm{dK}-3$ series, and adds two (wedge $\mathrm{dK}-3$ ) or three (triangle $\mathrm{dK}-3$ ) edges in the graph.

\section{RELATED WORK}

For sharing social network data, naive ID removal and $\mathrm{K}$ anonymity are two widely used methods in social network data anonymization [7, 12, 13]. Recently, researchers proposed various kinds of structural-based de-anonymization attacks $[4,8,9,11]$. They revealed the vulnerabilities of previous anonymization techniques in different angles. Differential privacy, which could achieve a strong privacy guarantee, was initially proposed to release certain data mining results, like the degree distribution and other graph patterns [2].

\section{CONCLUSION}

In this paper, we propose a uniform scheme that combines three levels of $\mathrm{dK}$ graph models to publish a perturbed social network. We design two different sub-schemes, CAT and LTH, to regenerate the graph and reduce the error under the differential privacy noise impact. Our two schemes have different merits in preserving graph utility. The design, analysis, and comparison also reveal more insights and challenges in using multiple levels of graph abstraction models together in differential private graph releasing for OSNs.

\section{REFERENCES}

[1] Charo I Del Genio, Hyunju Kim, Zoltán Toroczkai, and Kevin E Bassler. Efficient and exact sampling of simple graphs with given arbitrary degree sequence. PloS one, 5(4):e10012, 2010.

[2] Cynthia Dwork. Differential privacy. In Encyclopedia of Cryptography and Security, pages 338-340. Springer, 2011.

[3] Cynthia Dwork, Frank McSherry, Kobbi Nissim, and Adam Smith. Calibrating noise to sensitivity in private data analysis. In Theory of cryptography conference, pages 265-284. Springer, 2006.

[4] Shouling Ji, Weiqing Li, Shukun Yang, Prateek Mittal, and Raheem Beyah. On the relative de-anonymizability of graph data: Quantification and evaluation. 2016

[5] Priya Mahadevan, Dmitri Krioukov, Kevin Fall, and Amin Vahdat. Systematic topology analysis and generation using degree correlations. In ACM SIGCOMM Computer Communication Review, volume 36, pages 135-146. ACM, 2006.

[6] Frank McSherry and Kunal Talwar. Mechanism design via differential privacy. In Foundations of Computer Science, 2007. FOCS'07. 48th Annual IEEE Symposium on, pages 94-103. IEEE, 2007.

[7] Arvind Narayanan and Vitaly Shmatikov. De-anonymizing social networks. In Security and Privacy, 2009 30th IEEE Symposium on, pages 173-187. IEEE, 2009.

[8] Wei Peng, Feng Li, Xukai Zou, and Jie Wu. Seed and grow: An attack against anonymized social networks. In Sensor, Mesh and Ad Hoc Communications and Networks (SECON), 2012 9th Annual IEEE Communications Society Conference on, pages 587-595. IEEE, 2012.

[9] Jianwei Qian, Xiang-Yang Li, Chunhong Zhang, and Linlin Chen. De-anonymizing social networks and inferring private attributes using knowledge graphs. 2016.

[10] Alessandra Sala, Xiaohan Zhao, Christo Wilson, Haitao Zheng, and Ben Y Zhao. Sharing graphs using differentially private graph models. In Proceedings of the 2011 ACM SIGCOMM conference on Internet measurement conference, pages 81-98. ACM, 2011.

[11] Guojun Wang, Qin Liu, Feng Li, Shuhui Yang, and Jie Wu. Outsourcing privacy-preserving social networks to a cloud. In INFOCOM, 2013 Proceedings IEEE, pages 2886-2894. IEEE, 2013.

[12] Bin Zhou and Jian Pei. Preserving privacy in social networks against neighborhood attacks. In Data Engineering, 2008. ICDE 2008. IEEE 24th International Conference on, pages 506-515. IEEE, 2008.

[13] Lei Zou, Lei Chen, and M Tamer Özsu. K-automorphism: A general framework for privacy preserving network publication. Proceedings of the VLDB Endowment, 2(1):946-957, 2009. 